\title{
Nasopharyngeal biopsy in adults: a large-scale study in a non endemic area*
}

\author{
Sara Abu-Ghanem', Narin-Nard Carmel', Gilad Horowitz', Moshe Yehuda', \\ Rhinology 53: 142-148, 2015 \\ Moshe Leshno², Yasmin Abu-Ghanem', Dan M. Fliss', Avraham Abergel' \\ DOl:10.4193/Rhino14.130 \\ *Received for publication: \\ ' Department of Otolaryngology Head and Neck Surgery and Maxillofacial Surgery, Tel-Aviv Sourasky Medical Center, Tel Aviv, Israel May 31 , 2014 \\ 2 Faculty of Management, Tel Aviv University, Tel-Aviv, Israel \\ Accepted: September 21, 2014
}

\begin{abstract}
Background: Limited data exist on the referral criteria for endoscopic-guided nasopharyngeal biopsy to rule out nasopharyngeal malignancy among adults in a non-endemic area.
\end{abstract}

Methods: Retrospective study of all adult patients that had been referred to our outpatient clinic to undergo endoscopic-guided nasopharyngeal biopsy to exclude nasopharyngeal malignancy between 1/2006-10/2013. All medical consultation referral letters were reviewed, and statistical analyses were conducted to evaluate clinically significant predictors (demographics, clinical manifestations, nasopharyngeal endoscopic findings) for nasopharyngeal malignancy.

Results: A total of 470 patients (470 nasopharyngeal biopsies, $54.9 \%$ males and $66 \%$ smokers) were included. The most common pathological result was adenoid/lymphoid hyperplasia (76.2\%). The overall negative rate of all biopsies for malignancy was $94.2 \%$. Twenty-seven patients had nasopharyngeal malignancy: 22 had squamous cell carcinoma and 5 had non-Hodgkin's lymphoma. Advanced age, cervical mass, and suspicious nasopharyngeal mass were independent risk factors for nasopharyngeal malignancy on univariate and multivariate analyses.

Conclusions: Nasopharyngeal biopsy may safely be avoided in adults living in a non-endemic area for NPC who are free of the risk factors of advanced age, the presence of a cervical mass, and suspicious nasopharyngeal mass.

Key words: nasopharyngeal carcinoma, neck mass, presentation, biopsy, endoscopy

\section{Introduction}

Nasopharyngeal carcinoma (NPC) is a malignant squamous cell carcinoma (SCC) tumour arising from the mucosal epithelium of the nasopharynx. There is marked geographical variation in the incidence of NPC. It is relatively rare in most parts of the world, accounting for $2 \%$ of all head and neck SCCs and $\sim 0.25 \%$ of all cancers, with an incidence of 0.5-2 per 100,000 in Western Europe and the US ${ }^{(1)}$. In contrast, the disease is endemic in southern China, northern Africa, and Alaska, where the incidence may reach 25 cases per 100,000 per year ${ }^{(1-5)}$. Israel is considered to be a low-to-intermediate incidence area of NPC, although the rate among immigrants from North Africa is relatively higher ${ }^{(6,7)}$. Multiple risk factors have been suggested to be associated with the risk of developing NPC, including male gender, Chinese (or Asian) ancestry, Epstein-Barr virus exposure, heavy alcohol in- take, first-degree relative with NPC, occupational exposures and environmental exposures ${ }^{(7,8)}$. Interestingly, unlike other SSCs of the head and neck, the role of cigarette smoking is controversial (7).

Virtually all NPC patients are symptomatic at the time of diagnosis, with fewer than $1 \%$ of NPC patients being asymptomatic and diagnosed incidentally. In the majority of cases, the clinical presentation includes the presence of an asymptomatic cervical neck mass typically localized to the posterior cervical triangle or the superior jugular nodal chain, with additional clinical signs and symptoms that include nasal obstruction, nasal discharge, epistaxis, serous otitis media (SOM), otalgia, hearing loss, and headache ${ }^{(9-16)}$. In their review published in Lancet in 2005, Wei and Sham divided the symptoms of NPC patients into four 
categories: 1) caused by the presence of a tumour mass in the nasopharynx (epistaxis, nasal obstruction, and discharge), 2) associated with dysfunction of the eustachian tube (SOM/hearing loss), 3) associated with the superior extension of the tumour (headache, diplopia, facial pain, and numbness), and 4) the presence of a palpable neck mass ${ }^{(4)}$.

Sinonasal non-Hodgkin's lymphoma (NHL) is a rare form of extranodal lymphoma in the Western population, representing less than $0.5 \%$ of all cases ${ }^{(17)}$. Primary NHL of the nasopharynx represents $10-28 \%$ of Waldeyer's ring lymphomas ${ }^{(18,19)}$. These lymphomas predominantly occur in males between $50-60$ years of age and share similar signs and symptoms as SCC of the nasopharynx, including cervical adenopathy, epistaxis, nasal obstruction and/or decreased hearing ${ }^{(17,19,20)}$. In some cases, presentation can include B symptoms, such as fever and weight loss ${ }^{(20)}$.

It has been suggested that early diagnosis may improve the prognosis of this aggressive disease ${ }^{(17)}$. Treatment results for nasopharyngeal malignancy are not satisfactory in the advanced stage, and early diagnosis and appropriate management are important to achieve favorable treatment results. The conventional method of diagnosing nasopharyngeal malignancy is by clinical examination and biopsy guided by fiberoptic nasopharyngeal endoscopy (nasopharyngoscopy). However, there are no clear indications when to perform a nasopharyngeal biopsy. A suspicious nasopharyngeal mass, epistaxis, and neck mass are common manifestations, and many others have shown there significance ${ }^{(21)}$. In contrast, there are only a few reports on the role of other clinical presentations and nasopharyngoscopy findings to justify such biopsies in adults, especially among adults in low-endemic areas.

Our institution is a tertiary referral center for endoscopic-guided nasopharyngeal biopsy and histological diagnosis. It is located in a low-endemic area for nasopharyngeal malignancy. It was our impression that the criteria for referring a patient for nasopharyngeal biopsy to exclude a suspected nasopharyngeal malignancy (including nasopharyngoscopy findings and clinical manifestations) were more numerous than those compiled in Wei and Sham's comprehensive review ${ }^{(4)}$. Moreover, there are no published reports on the criteria for performing these biopsies in low-endemic areas, nor on the role of clinical manifestations and nasopharyngoscopy findings, alone or in combination, in the decision-making process to perform one. The objectives of the current study are to 1) evaluate the safety and indications for an office-based fiberoptic nasopharyngeal biopsy, and 2) to identify significant predictors for positive nasopharyngeal biopsies in patients with no history of NPC in a low-endemic area for NPC.

\section{Materials and methods}

Study design, setting and study population

Appropriate institutional review board approval was obtained for this retrospective review of the clinical records of all adults aged over 17 years who underwent fiberoptic guided nasopharyngeal biopsy to rule out nasopharyngeal malignancy between January 2006 and October 2013 in our tertiary referral center. Exclusion criteria included adults with known nasopharyngeal malignancy and those with missing pertinent data. All patients included in the current study were referred to our outpatient clinic by a community otolaryngologist to undergo a nasopharyngeal biopsy to rule out nasopharyngeal malignancy. All medical consultation referral letters were reviewed, and data on selected demographics (age, gender, smoking status), clinical manifestations (presenting symptoms and signs), and clinical findings on fiberoptic nasopharyngoscopy were extracted. Pathological reports were reviewed for biopsy results. Clinical manifestations (presenting signs and symptoms) were grouped as follows: 1) otitis media with effusion (OME)/conductive hearing loss $(\mathrm{CHL})$ based on otoscopy and audiometric examinations; 2) nasal symptoms (nasal congestion/obstruction/ discharge or snoring); 3) aural fullness with normal otoscopy and normal audiometric examination; 4) an incidental nasopharyngeal radiological finding on computed tomography $(\mathrm{CT}) / \mathrm{mag}$ netic resonance imaging (MRI) scans (e.g., a CT scan following head trauma) or an incidental nasopharyngeal finding during a routine physical examination or for other indications (e.g., prior to thyroid surgery); 5) blood-streaked sputum, bloody nasal discharge, or frank epistaxis; 6) laryngeal complaints, including sore throat, coughing, hoarseness, postnasal drip and dysphagia; 7) cervical lymphadenopathy; 8 ) headache, diplopia, facial pain, and facial numbness. Fiberoptic nasopharyngoscopy findings were grouped as follows: 1) normal tissue with no visible mass; 2) adenoidal (hypertrophic lymphoid tissue) appearance; 3) suspicious mass (i.e., either irregular, granular or exophytic mucosa); 4) nasopharyngeal mass with cystic appearance.

All nasopharyngeal biopsies were performed under topical anesthesia using neurosurgical patties soaked with tetracaine $4 \%$ and oxymetazoline hydrochloride $0.05 \%$. A $4 \mathrm{~mm} 0^{\circ}$ rigid endoscope was introduced through the inferior meatus (Storz, Tuttlingen, Germany). A good view of the nasal cavity and the nasopharynx was possible in all cases, and biopsy was performed using a punch forceps. All biopsy specimens were immersed in formalin and then embedded in paraffin. Sections cut from the paraffin blocks were stained with hematoxylin and eosin.

\section{Statistical analysis}

Statistical analyses were performed with SAS system software package version 9.2 (SAS, Cary, NC, USA). The numeric variables 
Table 1. Comparison of the demographic characteristics between the no nasopharyngeal malignancy and the nasopharyngeal malignancy groups based on biopsy findings.

\begin{tabular}{|c|c|c|}
\hline \multicolumn{3}{|c|}{ Characteristic } \\
\hline Age & Median \pm SD, years & Range, years \\
\hline Overall & $42 \pm 15.8$ & $18-92$ \\
\hline No malignancy & $43.2 \pm 15.4$ & $18-88$ \\
\hline Malignancy & $59.3 \pm 15.6$ & $22-92$ \\
\hline Gender & Male & Female \\
\hline Overall & $258 / 470$ (54.9\%) & $212 / 470(45.1 \%)$ \\
\hline No malignancy & $243 / 443(54.8 \%)$ & $200 / 443(45.2 \%)$ \\
\hline Malignancy & $15 / 27(55.6 \%)$ & $12 / 27(44.4 \%)$ \\
\hline Smoking status & Non-smoker & Smoker \\
\hline Overall & $72 / 214(33.6 \%)$ & $142 / 214(66.3 \%)$ \\
\hline No malignancy & $67 / 196$ (34.2\%) & $129 / 196(65.8 \%)$ \\
\hline Malignancy & $5 / 18(27.8 \%)$ & $13 / 18(72.2 \%)$ \\
\hline
\end{tabular}

$\mathrm{SD}=$ standard deviation

were presented as total numbers, percentages, and mean \pm standard deviation (SD) values. The patients were divided into two groups based on the pathology results of the nasopharyngeal biopsy: 1) the no malignancy group, which included patients with adenoid/ lymphoid hyperplasia, normal mucosa, or Tornwaldt's cyst, and 2) the malignancy group, which included patients with nasopharyngeal SCC or lymphoproliferative disorder. The $\chi^{2}$ test or Fisher's exact test were applied to compare those two groups for demographics, clinical manifestations, and fiberoptic nasopharyngoscopy findings. Univariate and multivariate logistic regression analyses were performed to identify significant risk factors (demographic variables, clinical manifestations, nasopharyngoscopy findings) for nasopharyngeal biopsies positive for malignancy. Odds ratios (OR) and their 95\% confidence interval $(\mathrm{Cl})$ were calculated using univariate logistic regression with the Firth's penalized maximum likelihood estimation to reduce bias in the parameter estimates. This method is useful in cases of the separability that often occurs when the event is rare. Statistical significance was determined as $p<0.05$. Multivariate logistic regression analysis was used to develop the nomogram. Specifically, stepwise logistic regression analysis was applied to determine which factors were independent and significant predictors of nasopharyngeal malignancy in the model building set. A nomogram for a positive nasopharyngeal biopsy for malignancy was developed from the final logistic regression model findings.

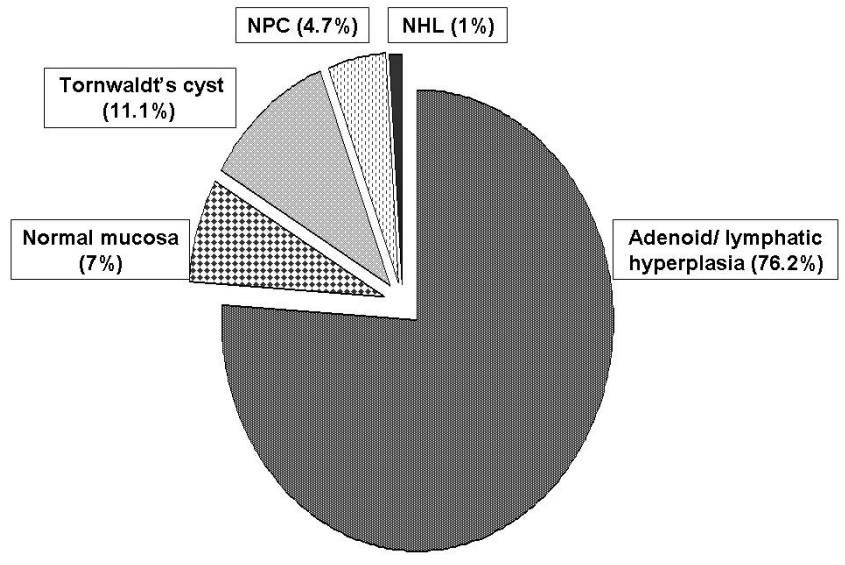

Figure 1. Distribution by percentages of the pathology results of 470 nasopharyngeal biopsies. NPC= nasopharyngeal carcinoma; $\mathrm{NHL}$, nonHodgkin's lymphoma.

\section{Results}

Six-hundred and twenty-one nasopharyngeal biopsies were performed during the study period, of which 470 fulfilled the above-cited study inclusion criteria and underwent analysis. The biopsy was well tolerated by all patients, and there were no complications. There were 258 males (54.9\%) and 212 females (45.1\%) whose median age at the time of the procedure was 42 years (range, 18-92 years, SD 15.87 years). Data on smoking status were available for 214 of the patients, and 142 (66.6\%) of them were smokers.

The subjects were divided into two groups based on the pathological results: 1 ) the malignancy group included 27 subjects (15 males, 12 females; median age [SD], 59.3 [15.6] years; range, 2292 years) with 27 biopsies ( 22 newly diagnosed SCC and 5 newly diagnosed B-cell non-Hodgkin's lymphoma), and 2) the no malignancy group included 443 subjects ( 243 males, 200 females; median age [SD], 43.2 [15.4] years; range, 18-88 years) with 443 biopsies. Table 1 summarizes the demographic data of the two study groups. As determined by the pathology results, the negative rate of all nasopharyngeal biopsies in this study was $94.2 \%$ (443/470). The majority of the biopsies revealed adenoids/lymphoid hyperplasias (358/470, 76.2\%) (Figure 1). The prevalence of clinical manifestations and nasopharyngoscopy findings in the two study groups are shown in Figures 2 and 3, respectively. No patient presented with cranial nerve impairment. The majority of patients that were referred for nasopharyngeal biopsy were presented with OME/CHL (135/470): 98 had unilateral and 37 had bilateral OME/CHL. Only eight of them were found with nasopharyngeal malignancy $(8 / 135,5.9 \%)$. No significant difference was found between the malignancy and no malignancy groups with respect to OME/CHL laterality: $72.4 \%$ vs $75 \%$ for 
Table 2. Univariate and multivariate analyses of significant predictors for nasopharyngeal malignancy.

\begin{tabular}{lccccccc} 
& \multicolumn{2}{c}{ Univariate analysis } & \multicolumn{3}{c}{ Multivariate analysis } \\
& OR & $95 \% \mathrm{Cl}$ & p value & OR & $95 \%$ Cl & p value \\
\hline Age & 1.064 & $1.037-1.096$ & $<0.0001$ & 1.074 & $1.026-1.124$ & 0.0022 \\
Cervical mass & 13.96 & $5.9-13.1$ & $<0.0001$ & 19.47 & $3.7-102.4$ & 0.0005 \\
\hline Suspicious NPX mass & 135.7 & $42.5-433.9$ & $<0.0001$ & 78.9 & $21.3-292.5$ & $<0.0001$ \\
Headaches & 4.13 & $1.1-15.5$ & 0.02 & & &
\end{tabular}

$\mathrm{OR}=$ odds ratio; $\mathrm{Cl}=$ confidence interval $\mathrm{NPX}=$ nasopharyngeal

unilateral $\mathrm{OME} / \mathrm{CHL}$ and $27.6 \%$ vs $25 \%$ for bilateral $\mathrm{OME} / \mathrm{CHL}$, respectively $(p>0.05)$.

The most common clinical manifestation in the malignancy group was a cervical mass $(12 / 27,44.4 \%)$, followed by $\mathrm{CHL}(8 / 27$, $29.7 \%)$, headaches $(3 / 27,11.1 \%)$, nasal symptoms $(2 / 27,7.4 \%)$, and bloody sputum/epistaxis (2/27, 7.4\%) (Figure 2). None of the referred patients who had aural fullness sensation with normal otoscopy, laryngeal complaints or an incidental nasopharyngeal finding had malignancy on biopsy (Figure 2). The most common nasopharyngoscopic finding in the malignancy group was a suspicious nasopharyngeal mass $(23 / 27,85.2 \%)$ (Figure 3). None of the patients that were referred with a normal-appearing nasopharynx on endoscopy were found to have malignancy on biopsy (Figure 3).
Univariate logistic regression analyses revealed four significant risk factors for malignancy: age $(\mathrm{OR}, 1.064 ; 95 \% \mathrm{Cl}, 1.037$ 1.096; $\mathrm{p}<0.0001)$, cervical mass (OR, 13.96; $95 \% \mathrm{Cl}, 5.9-13.1$; $\mathrm{p}<0.0001)$, headaches $(\mathrm{OR}, 4.13 ; 95 \% \mathrm{Cl}, 1.1-15.5 ; 0.02, \mathrm{p}=$ 0.02 ), and suspicious nasopharyngeal mass (OR, 135.7; $95 \% \mathrm{Cl}$, 42.5-433.9; $\mathrm{p}<0.0001)$. The presence of adenoidal/lymphoid tissue was a negative predictor for malignancy (OR, 0.04; $95 \% \mathrm{Cl}$, 0.0094-0.17; $\mathrm{p}<0.0001$ ). Multivariate logistic regression analysis showed significant differences between the malignancy and non-malignancy groups only in age $(\mathrm{OR}, 1.074 ; 95 \% \mathrm{Cl}, 1.026$ 1.124; $\mathrm{p}=0.0022)$, a cervical mass (OR, 19.47; 95\% Cl, 3.7-102.4; $\mathrm{p}=0.0005)$, and suspicious nasopharyngeal mass (OR, 78.9; $95 \%$ Cl, 21.3-292.5; $\mathrm{p}<0.0001$ ) (Table 2).

A nomogram was developed using the three independent risk

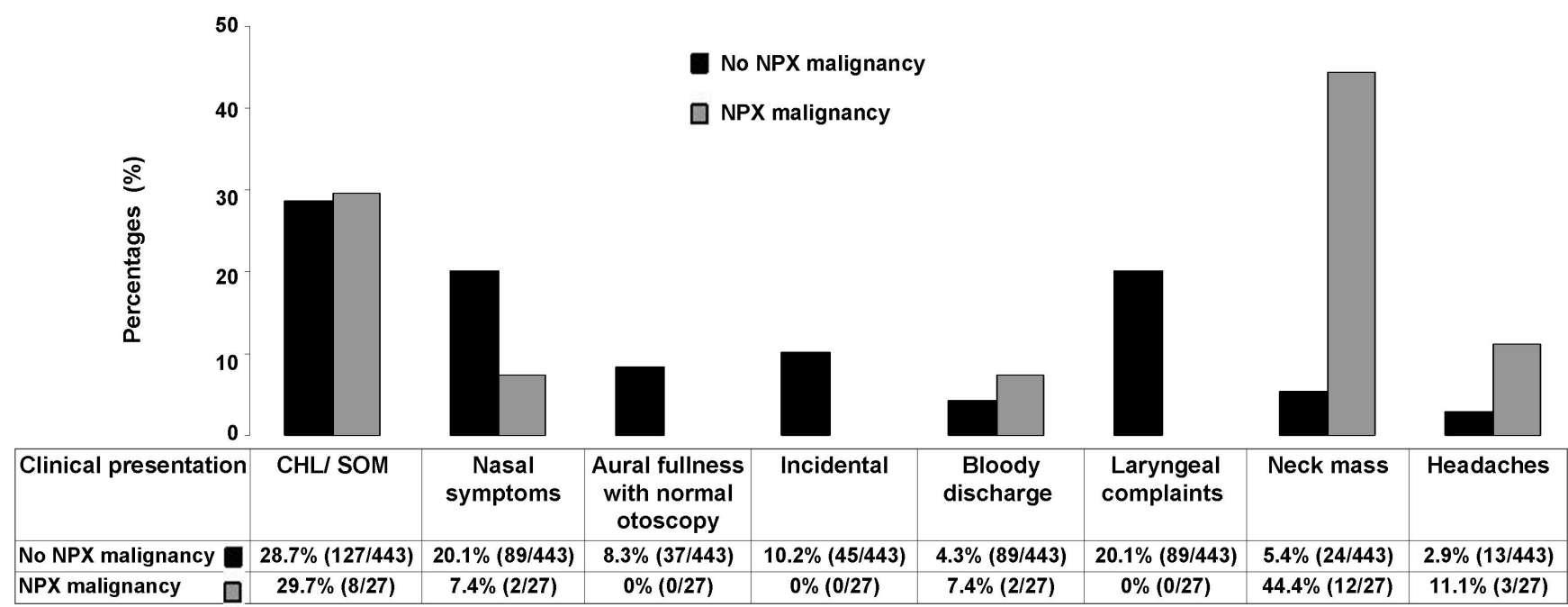

Figure 2. Comparison of the clinical manifestations between noncancerous (no nasopharyngeal malignancy) and cancerous (nasopharyngeal malignancy) groups based on biopsy results. NPX = nasopharyngeal; $\mathrm{CHL}=$ conductive hearing loss; SOM= serous otitis media. 


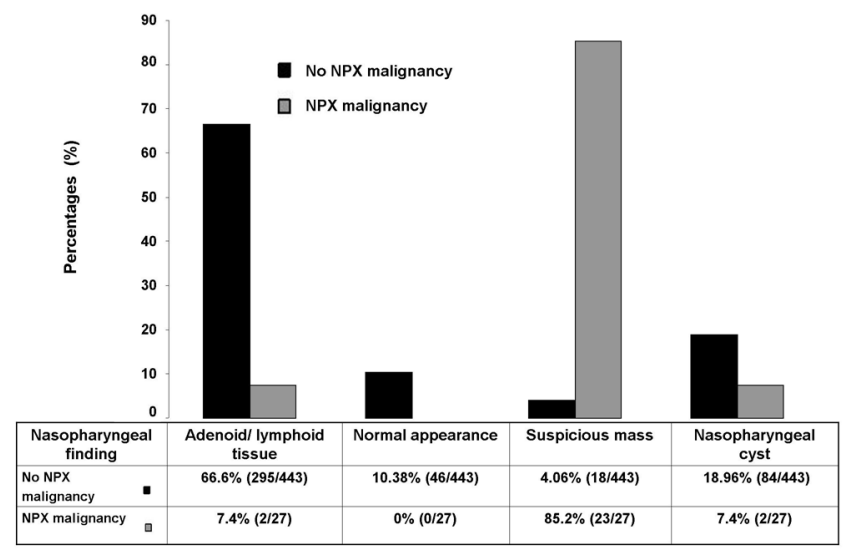

Figure 3. Comparison of the nasopharyngoscopic findings between noncancerous (no nasopharyngeal malignancy) and cancerous (nasopharyngeal malignancy) groups based on biopsy results. NPX = nasopharyngeal.

factors that had been found in the multivariate analysis to diagnose nasopharyngeal malignancy (Fig. 4). For each of the three significant predictors, an individual summed to calculate the overall likelihood of a positive biopsy, as described previously (22). For example, a 70-year-old male with a suspicious nasopharyngeal mass and no cervical mass had a risk of $40 \%$ to have a biopsy positive for nasopharyngeal malignancy according to the model (Figure 4).

\section{Discussion}

The anatomy of the nasopharynx explains the diverse clinical presentations of various pathologies and it is no surprise that patients with tumours in this location find their way not only to otolaryngologists but also to neurologists, ophthalmologists and general surgeons. The detection of nasopharyngeal malignancy in an early stage is often difficult because the symptoms are often subtle and nonspecific, causing a delay in the diagnosis and resulting in clinical presentation at an already advanced stage of disease ${ }^{(9)}$. It has been established that delay in diagnosis correlates with the degree of invasion and advancement of the disease stage ${ }^{(16,23)}$. Therefore, the importance of early detection cannot be over-emphasized: not only do patients with early therapeutic intervention have a much greater chance of cure, but they can also be spared the financial burden and associated toxicities of additional chemotherapy ${ }^{(24,25)}$.

A nasopharyngeal malignancy's clinical presentation is similar regardless of the histological type. For that reason and due to the small numbers of patients found with either NPC or NHL, we divided the patients into two groups based on the pathology results, one of SCC and NHL $(5.7 \%, 27 / 470)$ and the other of
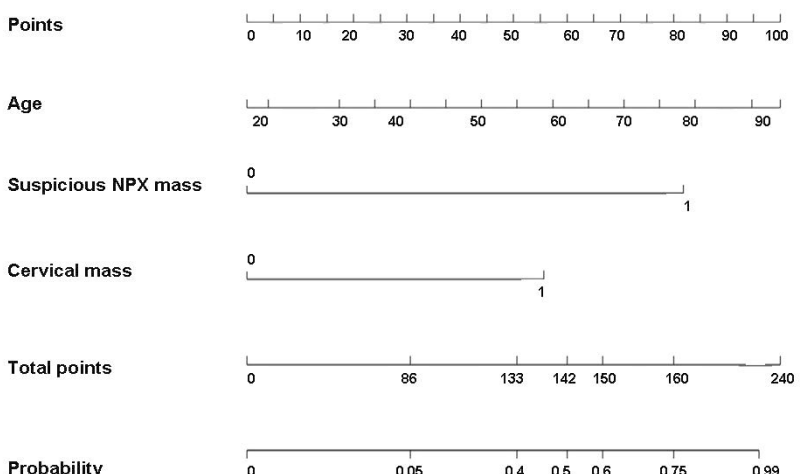

눈
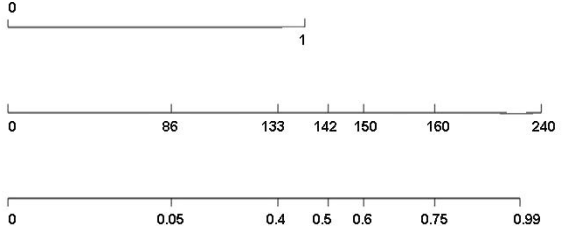

Figure 4. Severity profile of symptoms when grouped by symptom type. The percent of subjects who self-reported one or more symptoms within the symptom group (Overall; open blocks) and symptoms of moderate, severe, or very severe intensity (filled blocks). Secretory symptoms include: runny nose, sneezing and watery eyes. Pain/feverish/inflammatory symptoms include: sore/scratchy throat, headache, muscle aches and pains, feverishness and chilliness. Nasal congestion and cough are represented as individual symptoms.

nonmalignant adenoid/ lymphoid hyperplasia, normal mucosa, or Tornwaldt's cyst $(94.3 \%, 443 / 470)$.

Diagnosis of a nasopharyngeal malignancy is made by an endoscopic examination followed by biopsy of the suspected tumour. The latest edition of the National Comprehensive Cancer Network guidelines included detailed workup guidelines for the diagnosis and evaluation of nasopharyngeal carcinoma ${ }^{(26)}$. However, no clear indications could be found regarding when to perform a nasopharyngeal biopsy. More than 600 patients were referred to our tertiary outpatient clinic for endoscopic-guided biopsy in our over 6-year experience. Indeed, it is standard medical practice to examine and biopsy the nasopharynx, but the likelihood of detecting serious underlying pathology is probably small - the positive malignancy rate of all biopsies in the current study was only $5.7 \%$ (27/470). Our analysis revealed that nasopharyngeal malignancy was significantly associated with advanced age $(\mathrm{OR}, 1.074 ; 95 \% \mathrm{Cl}, 1.026-1.124 ; \mathrm{p}=0.0022)$, the presence of a cervical mass (OR, 19.47; $95 \% \mathrm{Cl}, 3.7-102.4 ; \mathrm{p}=$ $0.0005)$, and suspicious nasopharyngeal mass (OR, 78.9; $95 \% \mathrm{Cl}$, 21.3-292.5; $\mathrm{p}<0.0001)$.

There are only a few published studies on the role of routine nasopharyngeal biopsy in adults. One recent large-scale Taiwanese study examined the relationship between initial clinical manifestations and biopsy results ${ }^{(21)}$. The criteria for biopsy were more restricted than ours, and included nasopharyngeal tumours or 
an asymmetric nasopharynx, posterior nasal bleeding, aural symptoms, cranial nerve impairment, and neck masses of unknown origin. Moreover, that study also included patients with known nasopharyngeal carcinoma and involved a population from a high-endemic area for NPC (21).

Several reports examined the role of biopsy in patients presenting with isolated otitis media with effusion (OME). In the current study, of the 135 patients who were referred for nasopharyngeal biopsy with isolated $\mathrm{OME} / \mathrm{CHL}$, eight were diagnosed as having nasopharyngeal malignancy $(8 / 135,5.9 \%)$, and five of them were found to have a suspicious mass on their nasopharyngoscopy evaluation. Interestingly, CHL/OME was not a significant feature for nasopharyngeal malignancy in our current assessment (OR 1.04, 95\% Cl 0.47-2.45, p = 0.85). Moreover, when $\mathrm{OME} / \mathrm{CHL}$ laterality was tested, no significant difference was found between the malignancy and no malignancy groups. The available evidence in the literature suggests that a nasopharyngeal biopsy could be expected to yield a diagnosis of malignancy in $0.4 \%$ to $7.4 \%$ of all adults presenting with isolated $\mathrm{OME} / \mathrm{CHL}^{(27)}$. For example, Ho et al. found that the incidence of NPC among Taiwanese adults who appeared to suffer only from OME was 5.7\%; all of them had unilateral OME (5/87) (2). This study included only 3 patients with bilateral OME (3/87), therefore OME laterality could not be investigated as a predictor. Lower incidence rates were reported in studies carried out in low-endemic areas by Dempster et al. and Gaze et al. (0.4\% and $1.4 \%$, respectively) ${ }^{(28,29)}$. Those studies, however, did not include data on the nasopharyngoscopic findings. Glynn et al. reported that $4.7 \%(4 / 85)$ of the adults with CHL secondary to SOM had evidence of malignancy on nasopharyngeal biopsy: all of them had suspicious-looking masses on nasopharyngoscopy; three of them had unilateral OME and one had bilateral OME ${ }^{(30)}$. Therefore, all adult patients who present with "isolated" unilateral or bilateral middle ear effusion should undergo rigid or flexible nasopharyngoscopy. However, based on our results and others, nasopharyngeal biopsy is not mandatory in all cases, and there is a room for clinical judgment, especially in younger patients.

It is well accepted that it is not possible to exclude NPC by macroscopic examination of the nasopharynx alone, since nasopharyngeal tumours may spread submucosally, and that the nasopharynx may appear normal on flexible nasopharyngoscopy ${ }^{(31,32)}$. Therefore, if the clinical suspicion for nasopharyngeal malignancy is high, even if the suspected tumour cannot be visualized on endoscopic examination, other non-invasive techniques, such as MRI, should be used. In a recent publication, King et al. compared the accuracy of MRI with that of the current clinical standard of endoscopy and endoscopic biopsy (33). MRI had a sensitivity of $100 \%$ for cancer detection, helping to identify cancers missed at endoscopy and endoscopic biopsy and helping to identify patients who do not have NPC and who therefore do not need to undergo blind invasive sampling biopsies.

Patients who present with symptoms of NPC should be clinically assessed for physical signs of the disease. A definitive diagnosis of NPC requires a positive result of a biopsy taken from the tumour in the nasopharynx, supported either by its visualization in the nasopharynx or (in the case of predominantly submucosal tumours) its visualization with cross-sectional imaging. Although the current study supports the safety of office-based nasopharyngeal endoscopy with biopsy, it is an invasive and costly procedure that requires skill and should be carried out by otolaryngology specialists. We propose that tools, such as the nomogram presented in the current work, will be helpful in predicting the presence of malignancy and that they will considerably reduce the number of unnecessary biopsies and the associated stress and anxiety to the patient ${ }^{(34,35)}$.

\section{Conclusions}

To the best of our knowledge, this is the largest scale study that investigated the indications for nasopharyngeal biopsy and the association between initial clinical manifestations and nasopharyngeal endoscopic findings with positive biopsy for malignancy in a low endemic area for nasopharyngeal malignancy. Our data suggest that patients with none of the risk factors of advanced age, a cervical mass, and suspicious nasopharyngeal mass can be spared from undergoing invasive biopsy. The conclusions drawn from the results of the current study do not necessarily apply to a population where nasopharyngeal malignancies are endemic. However, it would appear that if no additional findings are detected on a careful history and clinical examination, and if a good view of the nasopharynx can be achieved with conventional fibreoptic examination, then the cost-effectiveness of the procedure should be seriously questioned. A larger sample size encompassing a greater number of patients and nasopharyngeal malignancy (SCC vs NHL) sub-groups, is required to confirm our study results, especially with regard to accuracy and riskanalysis information for individual patients.

\section{Authorship contribution}

NNC: Literature search, data collection; GH: Literature search, data collection; MY: Analysis of the results, critical review; ML: Planning the methods, analysis of the results, critical review; YAG: Planning the methods, data collection, analysis of the results; DMF: Analysis of the results, critical review; AA: Idea for research, planning the methods, data collection, analysis of the results, writing manuscript. 


\section{Acknowledgement}

Esther Eshkol, the institutional medical and scientific copyeditor, is thanked for editorial assistance.

\section{Conflicts of interest}

The authors have no funding, financial relationships, or conflicts of interest to disclose.

\section{References}

1. Ferlay J, Shin HR, Bray F, Forman D, Mathers C, Parkin DM. GLOBOCAN 2008 v2.0, Cancer Incidence and Mortality Worldwide: IARC CancerBase No. 10 [Internet]. Lyon, France: International Agency for Research on Cancer; 2010. Available from: http://globocan.iarc.fr.

2. Ho K-Y, Lee K-W, Chai C-Y, Kuo W-R, Wang $\mathrm{H}-\mathrm{M}$, Chien C-Y. Early recognition of nasopharyngeal cancer in adults with only otitis media with effusion. J Otolaryngol Head Neck Surg. 2008; 37: 362-365.

3. Yu MC, Yuan J-M. Epidemiology of nasopharyngeal carcinoma. Semin Cancer Biol. 2002; 12: 421-429.

4. Wei WI, Sham JST. Nasopharyngeal carcinoma. Lancet. 2005; 365: 2041-2054

5. Jemal A, Bray F, Center MM, Ferlay J, Ward E, Forman D. Global cancer statistics. CA Cancer J Clin. 2011; 61: 69-90.

6. Parkin DM, Iscovich J. Risk of cancer in migrants and their descendants in Israel: II. Carcinomas and germ-cell tumours. Int J Cancer 1997;70:654-660.

7. Chang ET, Adami H-O. The enigmatic epidemiology of nasopharyngeal carcinoma. Cancer Epidemiol Biomarkers Prev. 2006; 15: 1765-1777.

8. Wang Y, Zhang Y, Ma S. Racial differences in nasopharyngeal carcinoma in the United States. Cancer Epidemiol. 2013; 37: 793-802.

9. Wenig BM. Nasopharyngeal carcinoma. Ann Diagn Pathol. 1999; 3: 374-385.

10. Skinner DW, Van Hasselt CA, Tsao SY. Nasopharyngeal carcinoma: modes of presentation. Ann Otol Rhinol Laryngol. 1991; 100: 544-551.

11. August M, Dodson TB, Nastri A, Chuang SK. Nasopharyngeal carcinoma: clinical assessment and review of 176 cases. Oral Surg Oral Med Oral Pathol Oral Radiol Endod. 2001; 91: 205-214.

12. Erkal HS, Serin M, Cakmak A Nasopharyngeal carcinomas: analysis of patient, tumor and treatment characteristics determining outcome. Radiother Oncol. 2001; 61: 247-256.

13. Grammatica L, Achille G, Piepoli S, Paradiso A. Early, late symptoms and histological type of nasopharyngeal carcinoma. Oncol Rep. 2005;6:1395-1398.

14. Sun L-M, Epplein M, Li Cl, Vaughan TL, Weiss NS. Trends in the incidence rates of nasopharyngeal carcinoma among Chinese Americans living in Los Angeles County and the San Francisco metropolitan area, 19922002. Am J Epidemiol. 2005; 162: 1174-1178

15. Colaco RJ, Betts G, Donne A, et al. Nasopharyngeal carcinoma: a retrospec- tive review of demographics, treatment and patient outcome in a single centre. Clin Oncol (R Coll Radiol). 2013; 25: 171-177.

16. Yi J, Gao L, Huang X, et al. Nasopharyngeal carcinoma treated by radical radiotherapy alone: ten-year experience of a single institution. Int J Radiat Oncol Biol Phys. 2006; 65: 161-168.

17. Yen T-T, Wang R-C, Jiang R-S, Chen S-C, Wu S-H, Liang K-L. The diagnosis of sinonasal lymphoma: a challenge for rhinologists. Eur Arch Otorhinolaryngol. 2012; 269: 14631469.

18. Mohammadianpanah M, Ahmadloo N, Mozaffari MAN, Mosleh-Shirazi MA Omidvari S, Mosalaei A. Primary localized stages I and || non-Hodgkin's lymphoma of the nasopharynx: a retrospective 17-year single institutional experience. Ann Hematol. 2009; 88: 441-447.

19. Laskar S, Muckaden MA, Bahl G, et al. Primary non-Hodgkin's lymphoma of the nasopharynx: prognostic factors and outcome of 113 Indian patients. Leuk Lymphoma. 2006; 47: 2132-2139.

20. Kanumuri VV, Khan MN, Vazquez A, Govindaraj S, Baredes S, Eloy JA. Diffuse large B-cell lymphoma of the sinonasal tract: analysis of survival in 852 cases. Am J Otolaryngol. 2014; 35: 154-158.

21. Hsieh C-C, Wang W-H, Lin Y-C, Weng H-H, Lee K-F. A large-scale study of the association between biopsy results and clinical manifestations in patients with suspicion of nasopharyngeal carcinoma. Laryngoscope. 2012; 122: 1988-1993.

22. Suzuki H, Komiya A, Kamiya $N$, et al. Development of a nomogram to predict probability of positive initial prostate biopsy among Japanese patients. Urology. 2006; 67: 131-136

23. Sheng L, Shui Y, Shen L, Wei Q. Effect of patient-related delay in diagnosis on the extent of disease and prognosis in nasopharyngeal carcinoma. Am J Rhinol 2008; 22: 317-320.

24. Lee AWM, Ng WT, Chan YH, Sze H, Chan C, Lam TH. The battle against nasopharyngeal cancer. Radiother Oncol. 2012; 104: 272-278.

25. Neel HB, Taylor WF. New staging system for nasopharyngeal carcinoma. Long-term outcome. Arch Otolaryngol Head Neck Surg. 1989; 115: 1293-1303.

26. Pfister DG, Ang K-K, Brizel DM, et al. Head and neck cancers, version 2.2013. Featured updates to the NCCN guidelines. J Natl Compr Canc Netw. 2013; 11: 917-923.

27. Dang PT, Gubbels SP. Is nasopharyngoscopy necessary in adult-onset otitis media with effusion? Laryngoscope 2013; 123: 2081-
2082.

28. Dempster JH, Simpson DC. Nasopharyngeal neoplasms and their association with adult onset otitis media with effusion. Clin Otolaryngol Allied Sci. 1988; 13: 363-365.

29. Gaze MN, Keay DG, Smith IM, Hardcastle PF. Routine nasopharyngeal biopsy in adult secretory otitis media. Clin Otolaryngol Allied Sci 1992;17:183-184.

30. Glynn F, Keogh IJ, Ali TA, Timon Cl, Donnelly M. Routine nasopharyngeal biopsy in adults presenting with isolated serous otitis media: is it justified? J Laryngol Otol 2006;120:439-441.

31. Wei WI, Sham JST, Zong Y, Choy D, Ng MH. The efficacy of fiberoptic endoscopic examination and biopsy in the detection of early nasopharyngeal carcinoma. Cancer 1991; 67: 3127-3130.

32. Lee WC, Weiner GM, Campbell JB. Should nasopharyngeal biopsy be mandatory in adult unilateral glue ear? J Laryngol Otol. 1996; 110: 62-64.

33. King AD, Vlantis AC, Bhatia KSS, et al. Primary nasopharyngeal carcinoma: diagnostic accuracy of MR imaging versus that of endoscopy and endoscopic biopsy. Radiology. 2011; 258: 531-537.

34. Wade J, Rosario DJ, Macefield RC, et al. Psychological impact of prostate biopsy: physical symptoms, anxiety, and depression. J Clin Oncol. 2013; 31: 4235-4241.

35. Lang EV, Berbaum KS, Lutgendorf SK. Largecore breast biopsy: abnormal salivary cortisol profiles associated with uncertainty of diagnosis. Radiology 2009; 250: 631-637.

\author{
Avraham Abergel, MD \\ Department of Otolaryngology \\ Head and Neck Surgery and Maxillo- \\ facial Surgery \\ Tel Aviv Sourasky Medical Center \\ Sackler School of Medicine \\ Tel Aviv University \\ 6 Weizman Street \\ Tel Aviv 6423906 \\ Israel
}

Tel: +972-3-697 3573

Fax: +972-3-697 4840

E-mail:avrahamab@tlvmc.gov.il 